\title{
Stellar population and the origin of intra-cluster stars around brightest cluster galaxies: the case of NGC 3311 ${ }^{\star}$
}

\author{
L. Coccato ${ }^{1}$, O. Gerhard ${ }^{2}$, M. Arnaboldi ${ }^{1,3}$, and G. Ventimiglia ${ }^{2}$
}

\author{
${ }^{1}$ European Southern Observatory, Karl-Schwarzschild-Stra $\beta$ e 2, 85748 Garching bei München, Germany \\ e-mail: 1coccato@eso.org \\ 2 Max-Plank-Institut für Extraterrestrische Physik, Giessenbachstraße 1, 85741 Garching bei München, Germany \\ ${ }^{3}$ INAF, Osservatorio Astronomico di Pino Torinese, 10025 Pino Torinese, Italy
}

Received 23 June 2011 / Accepted 15 August 2011

\begin{abstract}
Context. We investigate the stellar population and the origin of diffuse light around brightest cluster galaxies.

Aims. We study the stellar population of the dynamically hot stellar halo of NGC 3311, the brightest galaxy in the Hydra I cluster, and that of photometric substructures in the diffuse light to constrain the origin of these components.

Methods. We analyze absorption lines in medium-resolution, long-slit spectra in the wavelength range 4800-5800 Å obtained with FORS2 at the Very Large Telescope. We measure the equivalent width of Lick indices out to $20 \mathrm{kpc}$ from the center of NGC 3311 and fit them with stellar population models that account for the $[\alpha / \mathrm{Fe}]$ overabundance.

Results. Stars in the dynamically hot halo of NGC 3311 are old (age $>13 \mathrm{Gyr})$, metal-poor $([\mathrm{Z} / \mathrm{H}] \sim-0.35$ ), and alpha-enhanced $([\alpha / \mathrm{Fe}] \sim 0.48)$. Together with the high velocity dispersion, these measurements indicate that the stars in the halo were accreted from the outskirts of other early-type galaxies, with a possible contribution from dwarf galaxies. We identify a region in the halo of NGC 3311 associated with a photometric substructure where the stellar population is even more metal-poor $([\mathrm{Z} / \mathrm{H}] \sim-0.73)$. In this region, our measurements are consistent with a composite stellar population superposed along the line of sight, consisting of stars from the dynamically hot halo of NGC 3311 and stars stripped from dwarf galaxies. The latter component contributes $\leq 28 \%$ to the local surface brightness.

Conclusions. The build-up of diffuse light around NGC 3311 is on-going. Based on the observed stellar population properties, the dominant part of these stars may have come from the outskirts of bright early-type galaxies, while stars from stripped dwarf galaxies are presently being added.
\end{abstract}

Key words. galaxies: halos - galaxies: clusters: individual: Hydra I (Abell 1060) - galaxies: individual: HCC 26

\section{Introduction}

Brightest cluster galaxies (BCGs) are the most massive and luminous objects located at the center of galaxy clusters. According to the hierarchical formation scenario, they assemble through the merger of smaller objects that are dragged into the cluster's center by dynamical friction (e.g. De Lucia \& Blaizot 2007). During the evolution of the cluster and the formation of the central BCG, stars are stripped from their host galaxies. Part of the stripped material is captured by the halos of the BCG and other massive galaxies, while the other part remains free-floating in the cluster potential, forming the so-called intra-cluster light (ICL). The details of the interplay between the formation of the BCG halos and the ICL are not yet fully understood. Observations of both components can give key information about the processes involved in the evolution of cluster galaxies.

In the last few years, progress was achieved in the characterization of the stellar population properties of the halos of BCGs at galactocentric radii larger than three effective radii by means of long-slit spectroscopy (Brough et al. 2007; Coccato et al. $2010 \mathrm{a}, \mathrm{b}$ ) or photometric studies of globular clusters (Forbes et al. 2011; Arnold et al. 2011). Stars in the BCG halos are generally old ( $\geq 10 \mathrm{Gyr})$, they have solar or sub-solar metallicity

$\star$ Based on observations collected at the European Southern Observatory for the program 082.A-0255(A). and super-solar $\alpha$-enhancement. Sometimes a change in the radial profiles of metallicity, $\alpha$-enhancement or color is observed, indicating that the stellar population properties in the outskirts are different from those in the central regions. The emerging interpretation is that the inner and outer regions formed in two separate phases: the central parts formed "in situ" (or through a single major merger), while the halo was accreted later through a series of minor mergers. This "two stages" formation process for BCGs is also supported by numerical simulations (e.g. Abadi et al. 2006; Oser et al. 2010) and photometric studies that compare the BCGs scaling relations between color gradients, velocity dispersions, and sizes with those of other early-type galaxies of similar mass or luminosity (Bernardi et al. 2011). Minor merger accretion in the second stage of this scenario is also consistent with the observed size evolution of early-type galaxies over redshift (Trujillo et al. 2007; van Dokkum et al. 2010), hydrodynamical cosmological simulations (Naab et al. 2009), and spectroscopic studies (Coccato et al. 2010b; Trujillo et al. 2011).

It is not clear yet whether the major contribution for the formation of the ICL comes from stars stripped in galaxy groups and subsequently captured by the cluster potential (Rudick et al. 2006, 2009; Kapferer et al. 2010), from stars of galaxies that interact with each other and with the cluster tidal field (Moore et al. 1996; Willman et al. 2004), from stars stripped from galaxies merging into the central BCG (Murante et al. 2007; Puchwein et al. 2010), or from entirely disrupted low surface brightness galaxies or dwarfs (Gnedin 2003). 
The connection between the accretion of the BCG halo and the formation of the ICL is hard to probe from an observational point of view, given the low surface brightness of these regions. Williams et al. (2007) measured the stellar population parameters for a sample of $\sim 5300$ intra-cluster stars in one field in the Virgo cluster. Although they found a heterogeneous stellar population, 70-80\% of the observed intra-cluster stars are old (age $>10 \mathrm{Gyr}$ ) and metal-poor (mean $[\mathrm{M} / \mathrm{H}] \sim-1.0$ ). On the other hand, other studies report super-solar metallicities in the diffuse light in Abell $3888\left(1.0 Z_{\odot}<Z<2.5 Z_{\odot}\right.$, Krick et al. 2006) that are interpreted as ICL formed early with the collapse of the main cluster, unrelated to the BCG halos. From a kinematic point of view, the line-of-sight velocity distribution of intra-cluster planetary nebulae in one Coma cluster core field is consistent with the ICL being formed from stars stripped from the halos of the two brightest galaxy in the cluster while they orbit each other (Gerhard et al. 2007), while recent studies of absorption line spectra in the RX J0054.0-2823 cluster are consistent with the ICL being the remnant of tidally destroyed galaxies and streaming in the central regions of the cluster, in which the three central giant ellipticals act as "grinding machines" (Toledo et al. 2011). The origin of the ICL and its connection with the BCG halos is thus not fully understood, and it is likely that several mechanisms are acting in its formation.

In this context we have been carrying out a project aimed at studying the formation of the intra-cluster stellar component in the Hydra I galaxy cluster (Abell 1060), and this paper is the fourth in this series. We observed a rapid increase of the velocity dispersion radial profile in the halo of NGC 3311, the BCG in Hydra I (Ventimiglia et al. 2010). This was interpreted as caused by a dynamically hot stellar halo around NGC 3311, dominated by intra-cluster stars. We also observed the velocities of planetary nebulae in the core of the Hydra I cluster, the major component of which is consistent with being part of this halo of intra-cluster stars (Ventimiglia et al. 2011). The distribution and kinematics of the entire sample of intra-cluster planetary nebulae indicate that most of the diffuse light is still not phasemixed. Finally, Arnaboldi et al. (2011) found that the hot stellar halo is not centered on NGC 3311, and detected a photometric sub-structure (excess of light, hereafter) superposed on the intracluster halo. The contours of light that best approximate this photometric excess are shown in Fig. 1. Their results indicate that this excess of light has line-of-sight velocity $\sim 1200 \mathrm{~km} \mathrm{~s}^{-1}$ relative to the stellar halo around NGC 3311, and that it is related to one or more dwarf galaxies disrupted in this region. The build-up of the ICL and the dynamically hot stellar halo around NGC 3311 are therefore ongoing through the accretion of material from galaxies falling into the cluster core and tidally interacting with its potential well.

In this paper we investigate the nature of the dynamically hot stellar halo around NGC 3311 and the excess of light by studying their stellar populations. Section 2 presents the spectroscopic observations and the data reduction steps. Section 3 describes measurements of equivalent widths of the Lick indices and the derivation of stellar age, metallicity, and $\alpha$-enhancement. In Sect. 4, we discuss the origins of the stellar halo of NGC 3311 and the excess of light, and finally in Section 5 we conclude with a brief summary.

\section{Spectroscopic observations and data reduction}

The long-slit spectra were obtained during the nights of March 25-28 2009, with the focal reducer and low-dispersion spectrograph (FORS2, Appenzeller et al. 1998) mounted on the Unit

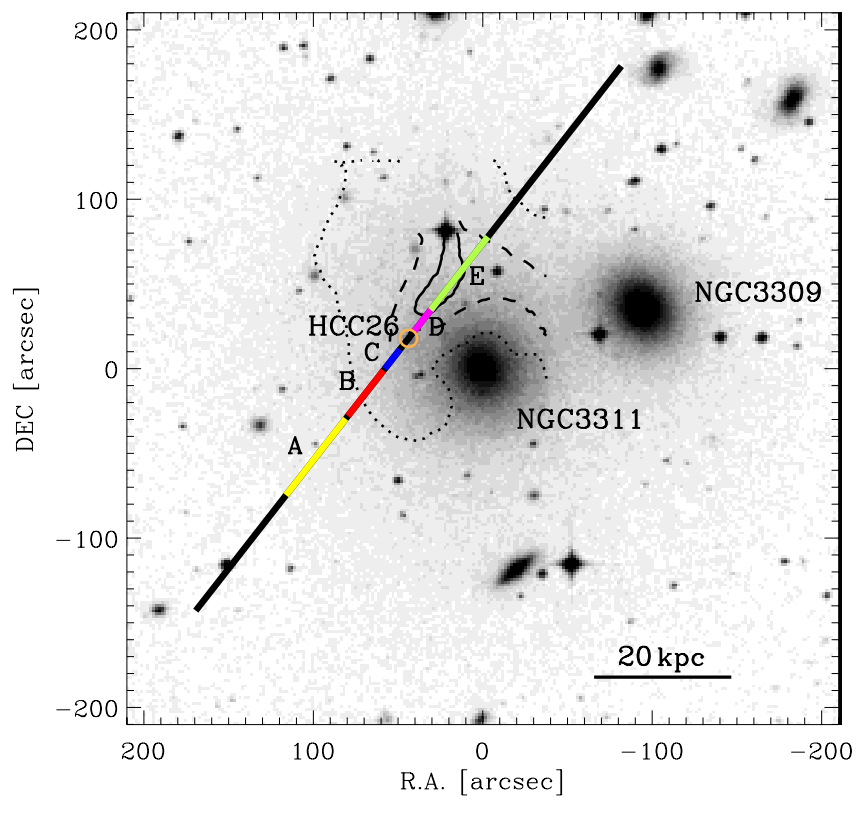

Fig. 1. Digital Sky Survey image of the central regions of the Hydra I cluster. The two brightest galaxies NGC 3311 and NGC 3309 are labeled. The thick black line represents the location of the FORS2 long slit. The orange circle marks the slit center on top of the dwarf galaxy HCC 26. The spectrum of HCC 26 was extracted from the central 6" (see text for details). The colored lines identify the location of the five slit sections (bins A-E) where we extracted the spectra of the halo of NGC 3311. Thin continuous, dashed and dotted lines represent approximately the contours of a photometric substructure found by Arnaboldi et al. (2011). We adopt a distance to NGC 3311 of $51 \mathrm{Mpc}$, from the NASA/IPAC extragalactic database.

Telescope 1 of the European Southern Observatory (ESO) in Paranal (Chile). The long-slit was oriented at position angle $\mathrm{PA}=142^{\circ}$, and centered on the dwarf galaxy HCC 26, which is $\sim 45^{\prime \prime}$ northeast from NGC 3311 (Fig. 1). The instrumental setup had the 1". 6 wide long-slit and the $1400 \mathrm{~V}+18$ grism with instrumental spectral resolution of $F W H M \sim 210 \mathrm{~km} \mathrm{~s}^{-1}$. The total exposure time was four hours. For more information on the instrumental set-up see Ventimiglia et al. (2010).

Data reduction (overscan and bias subtraction, flat-fielding correction, wavelength and flux calibrations) was performed with the FORS2 reduction pipeline version 3.0 under the ESOrex environment ${ }^{1}$ and IRAF $^{2}$ and IDL ad hoc scripts. The contribution from scattered light in the instrument was interpolated from the portions of the FORS2 detectors not illuminated by the long-slit (typically a few rows on the CCDs borders) and then subtracted. Scattered light amounted to $7 \pm 3 \%$ of the skybackground value, with negligible gradients along the dispersion direction. Wavelength calibration and spectral rectification were performed using $\mathrm{He}, \mathrm{Hg}+\mathrm{Cd}$, and $\mathrm{Ne}$ calibration lamp spectra and were cross-checked with the most intense emission lines in the sky spectrum. Errors on the wavelength calibration were $\sim 5 \mathrm{~km} \mathrm{~s}^{-1}$. Cosmic rays were removed using the FILTER/COSMIC routine in MIDAS ${ }^{3}$. Long-slit spectra on blank fields in the Hydra I cluster were also obtained close in time to the scientific spectra to evaluate the sky background and to correct for large-scale illumination patterns from the non-uniform

\footnotetext{
${ }^{1}$ Updated version of the FORS2 pipeline and ESOrex can be found at http://wWw . eso.org/sci/software/pipelines/

2 IRAF is distributed by NOAO, which is operated by AURA Inc., under contract with the National Science Foundation.

3 MIDAS is developed and maintained by ESO.
} 
illumination along the slit. All spectra were corrected for atmospheric extinction and flux-calibrated using spectro-photometric standard stars observed during twilight as part of the FORS2 standard calibration plan.

\subsection{Sky subtraction}

Accurate sky subtraction is important when measuring spectra of low surface brightness regions. To limit systematic effects as much as possible, we accounted for the sky-subtraction in two ways, following the strategy adopted in Coccato et al. (2010a). The mean flux of the continuum of the sky spectrum extracted from the outermost portions of the long slit was $\sim 25 \%$ higher than that of the sky spectrum extracted from blank fields in the Hydra I cluster. This indicated that the contributions from the halo of NGC 3311 and from intra-cluster stars were not negligible in the long-slit ends. We therefore decided to use the sky spectrum obtained from blank fields. Although they were taken close in time to the scientific spectra, they were not simultaneous. To minimize the effects of the variation in time of the intensity of the sky emission lines, we proceeded in a way similar to that of Davies (2007). For each night's sky spectrum, a fourth order polynomial was fitted to the continuum and then subtracted. The mean flux of the sky-background continuum in the $4800-5600 \AA$ range did not vary by more than $\pm 5 \%$ from night to night. The residual spectrum that contained emission and absorption line features was multiplied by a constant factor and added to the sky continuum previously subtracted. The constant factor (typically on the order of 1.20-1.30) was chosen to minimize the difference between the intensities of some selected sky emission lines measured in the scientific spectra and those measured in the sky spectra. The entire process was optimized in the wavelength range 4800-5800.

Finally, the sky-subtracted spectra were coadded using the center of HCC 26 as reference for alignment.

HCC 26. The spectrum of HCC 26 was extracted from the central 6 " of the FORS2 long-slit (orange circle in Fig. 1). The contribution of NGC 3311 was interpolated from the adjacent $\sim 5^{\prime \prime}$, 8, where residual flux of HCC26 is negligible (lower than $4 \%$ of the HCC 26 central value and comparable to the local noise level) and removed from the spectrum.

NGC 3311 halo. The spectrum of the halo of NGC 3311 was extracted along five sections of the FORS2 long-slit (bins A-E) from the sky-subtracted spectrum. Their locations are displayed in Fig. 1. The edges of the central bins C and D are $\sim 6^{\prime \prime}$ away from the slit center, at positions where the contamination from HCC 26 is lower than the background noise level. The sizes of the bins are chosen to ensure a minimal signal-to-noise ratio $\sim 25$ per spectral pixel in each bin, and to account for the CCD bad cosmetics and faint foreground stars in the slit. Outside the explored region the surface brightness level is too small to permit reliable measurements.

We quantified the residual contamination from sky emission lines on the final co-added science spectra to be lower than $2 \%$ of the subtracted sky itself, and lower than the noise level. This was determined in the following way:

1. We subtracted the best-fit models from the observed galaxy spectra, obtaining the residual spectra $R(\lambda)$ for each slit bin. We computed the average noise $\langle\mathrm{rms}\rangle$ values as standard deviations of the $R(\lambda)$ spectra in the wavelength range 4900$5550 \AA$.
2. We cross-correlated the $R(\lambda)$ with the continuum-subtracted sky spectrum $S_{\text {ems }}(\lambda)$ to detect evidence of contamination from residual emission lines. No significant correlation was found, meaning that the contamination was below the noise level.

3. We constructed a noise spectrum $N(\lambda)$ with mean 0 and standard deviation equal to the maximum $\langle\mathrm{rms}\rangle$, which is measured at the outermost slit section (bin A, Fig. 2).

4. We determined the minimum value of $K$ that ensured a significant correlation between the $S_{\text {ems }}(\lambda)$ spectra and $N(\lambda)+$ $K \cdot S_{\text {ems }}(\lambda)$. We found $K=0.02$, meaning that the highest possible contamination from residual sky-emission lines is $2 \%$ of the sky value. Indeed, if the contamination were higher, it would have been detected in step 2 . The $2 \%$ upper limit will be used in Sect. 3.1 to quantify the maximum systematic errors caused by residual sky contamination in the derived stellar population parameters.

\section{Line strength indices and single stellar population}

The spectra extracted from the slit sections were fitted with the MILES library of stellar template spectra (Sánchez-Blázquez et al. 2006) using the penalized pixel-fitting routine by Cappellari \& Emsellem (2004) to recover the stellar velocity $(V)$, velocity dispersion $(\sigma)$, third and fourth Gauss-Hermite moments ( $h 3$ and $h 4$ ) of the line-of-sight velocity distribution (LOSVD). In the fitting process, the spectra of the MILES library were convolved with a Gauss-Hermite function to match the instrumental FORS2 spectral resolution. The appropriate function was determined by fitting Gauss-Hermite moments to the correlation function between the spectra of one star observed during the run and its corresponding spectrum in the MILES library. The use of a Gauss-Hermite function instead of the commonly used Gaussian function ensured more reliable measurements, because the FORS2 instrumental line-spread function slightly deviates from a Gaussian profile. The extracted spectra are shown in Fig. 2 together with their best-fit models. We refer the reader to Ventimiglia et al. (2010) for the discussion of the kinematics. In this paper we focus on the measurements of the Lick indices and the derivation of the stellar population properties.

Galaxy spectra were set to the rest wavelength using the measured radial velocities and then broadened to match the Lick instrumental spectral resolution $(8.4 \AA$ at $5100 \AA$, Worthey \& Ottaviani 1997). The equivalent widths of the line strengths of several Lick indices were measured according to the definitions of Worthey et al. (1994), and their errors were evaluated using the empirical formulas by Cardiel et al. (1998).

The measurements were also corrected for kinematic broadening caused by the line-of-sight velocity distribution, using the multiplicative coefficients tabulated in Kuntschner (2004), which account for $\sigma, h 3$ and $h 4$. Because no coefficient is tabulated for the Fe5709, the corresponding factor was determined as the ratio of i) the intrinsic values measured on the optimal stellar template; and ii) the values measured on the optimal stellar template convolved with the measured line of sight velocity distribution.

A set of seven Lick standard stars were also observed with the same instrumental configuration. The line strengths of the Lick indices were measured for these stars and compared with the values tabulated in Worthey et al. (1994) to measure offsets to the Lick system. Small offsets were observed for the 

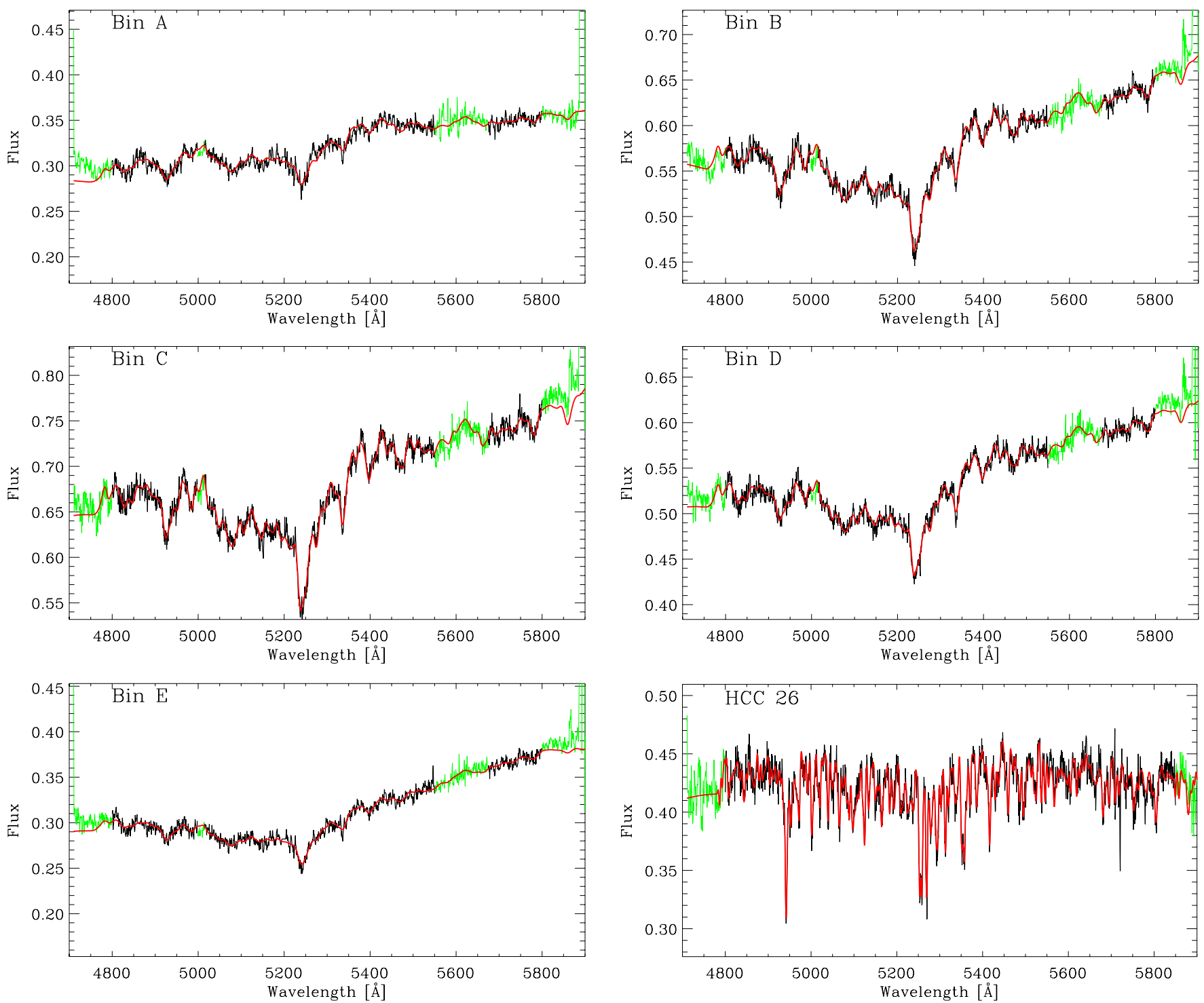

Fig. 2. Spectra extracted in the selected slit sections (bins A-E) and in the slit center (HCC 26). Black: observed spectrum, red: best-fit model, green wavelength region excluded from the fit.

$\mathrm{H} \beta(-0.11 \pm 0.08 \AA)$ and $\mathrm{Mg} b(-0.13 \pm 0.07 \AA)$ indices and were applied to our measurements.

Table 1 lists the final equivalent widths of the Lick indices we measured, corrected for kinematic broadening and offset to the Lick system. Errors in the equivalent widths also include the uncertainties in the offsets to the Lick system and in the correction for kinematic broadening.

\subsection{Luminosity-weighted single stellar population}

We compared our measured Lick indices $\mathrm{H} \beta, \mathrm{Mg} b$, Fe5270, Fe5335, Fe5105, Fe5406, and Fe5709 to the values predicted by the single stellar population models of Thomas et al. (2011). We interpolated the original grid of models so that we have steps of $\sim 0.4$ Gyr in age and $\sim 0.025$ dex in metallicity $([\mathrm{Z} / \mathrm{H}])$ and in $\alpha$-enhancement $([\alpha / \mathrm{Fe}])$. The best stellar population parameters are obtained by a $\chi^{2}$ minimization using the observed indices. Errors on the best-fit parameters are computed by means of Monte Carlo simulations.

To quantify the errors on age, $[\mathrm{Z} / \mathrm{H}]$ and $[\alpha / \mathrm{Fe}]$ caused by residual sky contamination in the spectra (see Sect. 2.1) we added to the scientific spectra an extra contribution $\pm 2 \%$ of the subtracted sky and measured the single stellar population parameters again. The best-fit luminosity weighted values of age, $[\mathrm{Z} / \mathrm{H}]$ and $[\alpha / \mathrm{Fe}]$ and the range allowed by potential contamination from sky emission lines are shown in Fig. 3 and listed in Table 2.

There are no data in the literature that extend from the center of NGC 3311 toward the halo and overlap with our measurements, but according to Loubser et al. (2008), the central region of $\mathrm{NGC} 3311$ is younger $\left(\mathrm{Age}_{0}=8.7 \pm 1.8 \mathrm{Gyr}\right.$ ) considerably more metal-rich $\left([\mathrm{Z} / \mathrm{H}]_{0}=0.12 \pm 0.07\right)$ than what we observe in the hot halo, and has similar abundance ratio $\left([\alpha / \mathrm{Fe}]_{0}=0.40 \pm 0.03\right)$.

\subsection{Contamination from foreground stars}

At $\sim 30^{\prime \prime}$ north of the center of bin $\mathrm{E}$ there is a bright star with $B \sim 12.2$ mag from the USNO-B1 catalog but unknown spectral classification. Although this star does not enter the slit directly (its distance to the long-slit is $\sim 20$ times the seeing FWHM), it is inside the FORS2 field of view and its light can be scattered 
Table 1. Line strengths of Lick indices in the hot stellar halo around NGC 3311.

\begin{tabular}{lccccccccr}
\hline \hline ID & $\begin{array}{c}\mathrm{PA} \\
{\left[{ }^{\prime}\right]}\end{array}$ & $\begin{array}{c}\mathrm{PA} \beta \\
{\left[{ }^{\circ}\right]}\end{array}$ & $\begin{array}{c}\mathrm{Fe} 5015 \\
{[\AA]}\end{array}$ & $\begin{array}{c}\mathrm{Mg} b \\
{[\AA]}\end{array}$ & $\begin{array}{c}\mathrm{Fe} 5270 \\
{[\AA]}\end{array}$ & $\begin{array}{c}\mathrm{Fe} 5335 \\
{[\AA]}\end{array}$ & $\begin{array}{c}\mathrm{Fe} 5406 \\
{[\AA]} \\
(\AA)\end{array}$ & $\begin{array}{c}\mathrm{Fe} 5709 \\
{[\AA]}\end{array}$ \\
$(1)$ & $(2)$ & $(3)$ & $(4)$ & $(5)$ & $(6)$ & $(7)$ & $(8)$ & $\begin{array}{c}{[\AA} \\
(9)\end{array}$ & $(10)$ \\
\hline $\mathrm{D}$ & 42.8 & 47.1 & $0.77 \pm 0.09$ & $3.14 \pm 0.19$ & $3.02 \pm 0.12$ & $1.63 \pm 0.10$ & $1.84 \pm 0.17$ & $1.13 \pm 0.09$ & $0.81 \pm 0.08$ \\
$\mathrm{C}$ & 50.2 & 83.7 & $0.96 \pm 0.09$ & $3.42 \pm 0.18$ & $3.41 \pm 0.10$ & $1.69 \pm 0.10$ & $2.05 \pm 0.15$ & $1.26 \pm 0.09$ & $0.85 \pm 0.08$ \\
$\mathrm{E}$ & 57.1 & 10.3 & $0.66 \pm 0.10$ & $2.57 \pm 0.22$ & $2.57 \pm 0.18$ & $1.24 \pm 0.11$ & $1.58 \pm 0.21$ & $0.77 \pm 0.12$ & $0.75 \pm 0.10$ \\
$\mathrm{~B}$ & 67.2 & 102.5 & $1.11 \pm 0.09$ & $3.40 \pm 0.19$ & $3.18 \pm 0.11$ & $1.78 \pm 0.10$ & $1.80 \pm 0.15$ & $1.23 \pm 0.09$ & $0.81 \pm 0.08$ \\
$\mathrm{~A}$ & 108.4 & 118.8 & $0.99 \pm 0.10$ & $4.05 \pm 0.24$ & $3.06 \pm 0.26$ & $1.68 \pm 0.18$ & $1.61 \pm 0.23$ & $0.77 \pm 0.13$ & $0.69 \pm 0.11$ \\
$\mathrm{HCC} 26$ & 45.0 & 64.0 & $1.85 \pm 0.09$ & $2.53 \pm 0.18$ & $1.85 \pm 0.07$ & $2.05 \pm 0.07$ & $1.87 \pm 0.13$ & $1.22 \pm 0.07$ & $0.61 \pm 0.09$ \\
\hline
\end{tabular}

Notes. Column 1: slit bin name, according to Fig. 1. Column 2: distance of the bin center from the center of NGC 3311. Column 3: position angle of the bin center with respect to the center of NGC 3311. North is $0^{\circ}$, East is $90^{\circ}$. Columns 4-11: equivalent widths of the Lick indices and their errors.

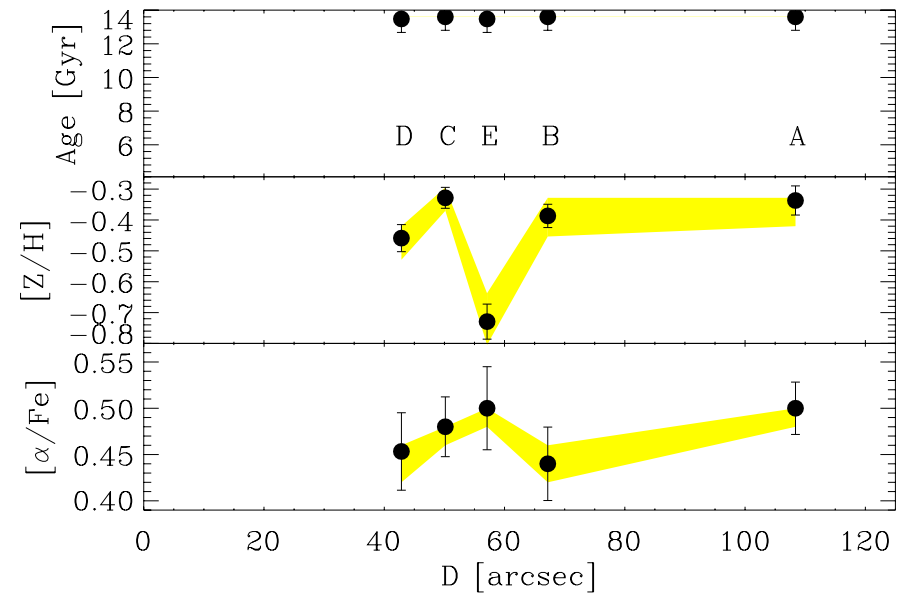

Fig. 3. Equivalent luminosity-weighted single stellar population values of age (upper panel), metallicity (central panel) and $\alpha$-enhancement (lower panel) obtained in the five long-slit sections, as function of the distance to NGC 3311. Labels in the upper panel identify each radial bin. The shaded yellow regions indicate the range of SSP parameters that would result if systematic residual effects in the sky subtraction at the level of $\leq \pm 2 \%$ were present (see Sect. 3.1 for details).

Table 2. Luminosity-weighted single stellar population parameters in the hot stellar halo around NGC 3311.

\begin{tabular}{lcccc}
\hline \hline ID & $a$ & Age & {$[\mathrm{Z} / \mathrm{H}]$} & {$[\alpha / \mathrm{Fe}]$} \\
& {$\left[{ }^{\prime \prime}\right]$} & {$[\mathrm{Gyr}]$} & & $(5)$ \\
\hline 1$)$ & $(2)$ & $(3)$ & $(4)$ & $(5)$ \\
\hline $\mathrm{D}$ & 43.2 & $13.5_{-0.8}^{+0.0}$ & $-0.46 \pm 0.04$ & $0.45 \pm 0.04$ \\
$\mathrm{C}$ & 50.8 & $13.5_{-0.8}^{+0.0}$ & $-0.33 \pm 0.03$ & $0.48 \pm 0.03$ \\
$\mathrm{E}$ & 61.3 & $13.5_{-0.8}^{+0.0}$ & $-0.73 \pm 0.06$ & $0.50 \pm 0.06$ \\
$\mathrm{~B}$ & 70.2 & $13.5_{-0.8}^{+0.0}$ & $-0.39 \pm 0.04$ & $0.44 \pm 0.04$ \\
$\mathrm{~A}$ & 117.0 & $13.5_{-0.8}^{+0.0}$ & $-0.34 \pm 0.05$ & $0.50 \pm 0.03$ \\
HCC 26 & 45.4 & $13.5_{-1.0}^{+0.0}$ & $-0.85 \pm 0.03$ & $-0.03 \pm 0.05$ \\
\hline
\end{tabular}

Notes. Column 1: bin name, according to Fig. 1. Column 2: distance of the bin center from the center of NGC 3311 projected onto the galaxy major axis assuming an axial ratio $b / a=0.89$ (Arnaboldi et al. 2011). Columns 3, 4: single stellar population best-fit values and their errors. $[\mathrm{Z} / \mathrm{H}]$ and $[\alpha / \mathrm{Fe}]$ are in logarithm of solar units.

along the optical path of the spectrograph and hit the detector. In Sect. 2 we quantified the amount of scattered light $(\sim 7 \pm 3 \%$ of the sky) from the portions of the detectors not covered by the long slit, and subtracted it from the scientific spectra. This ensures that the mean contribution from any scattered source is removed. The error in the quantification of the scattered light is very similar to the error in the sky subtraction, which does not explain the observed difference in stellar population properties (Fig. 3). Small residual variations of the scattered light intensity along the long-slit might still be present, but it is not possible to measure them. Nevertheless, because the light is scattered, we expect that it contaminates adjacent regions of the detector by a similar amount. On the other hand, we observe a large difference between the spectral properties of the adjacent bins D and E, suggesting that this difference reflects a variation in the stellar population properties in different slit sections.

\section{Discussion}

NGC 3311 is surrounded by a dynamically hot stellar halo, characterized by a rapid increase of the velocity dispersion of the stars (Hau et al. 2004; Loubser et al. 2008; Ventimiglia et al. 2010) and of the globular clusters system (Richtler et al. 2011). In this section we will use the information on the stellar population derived in Sect. 3.1 to constrain the origin of this dynamically hot stellar halo.

In this region, Arnaboldi et al. (2011) identified a photometric sub-structure (excess of light), which extends toward the northern and eastern sides of NGC 3311. In Fig. 1 we show the approximate contours of the excess of light in the field of view. An accurate determination of the morphology, photometry and precise isophotes of the excess is complicated by the presence of foreground stars and uncertainties in the background subtraction and is not the aim of the present paper. For our purposes it is enough to notice that the FORS2 long-slit crosses the excess of light from SE to NW, probing regions where its intensity is different. Although the isophotes in Fig. 1 are approximate, we expect that the contribution from the excess of light to the mean stellar population properties along the line of sight has a minimum in bin A and a maximum in bin E. From Fig. 3 we have evidence that the mean stellar populations in bins $E$ and D have different metallicities from those observed in the other slit sections. We interpret this difference as caused by the contamination from the excess of light, which is higher in bin $\mathrm{E}$ (where the difference in metallicity is more evident) and lower in bin D (where it is less evident).

We therefore split the discussion into two parts: Sect. 4.1 describes the stellar population properties inferred from the portion of the slit covered by bins A-C, while Sect. 4.2 describes those derived from bins D-E.

\subsection{The dynamically hot stellar halo around NGC 3311}

The spectra extracted in regions A-C contain the contribution mostly from stars in the dynamically hot halo around NGC 3311. 
Indeed, the rapid increase over radius of the velocity dispersion (see Sect. 4) suggests that the contribution of stars bound to the main body of NGC 3311 at these galactocentric distances is small. From the contour levels displayed in Fig. 1 we can conclude that the contribution from the excess of light is also small. Therefore, we consider the age, $[\mathrm{Z} / \mathrm{H}]$ and $[\alpha / \mathrm{Fe}]$ measured in bins $\mathrm{A}-\mathrm{C}$ as indicative of the mean luminosity-weighted stellar population properties in the dynamically hot halo around NGC 3311, dominated by intra-cluster stars.

In this region, stars are old (age $>13 \mathrm{Gyr}$ ), metal-poor $([\mathrm{Z} / \mathrm{H}]=-0.35 \pm 0.05)$ and formed over a rapid time scale $(<1 \mathrm{Gyr})$, as inferred from the high abundance ratio of $\alpha$ elements $([\alpha / \mathrm{Fe}]=0.48 \pm 0.03)$. Similar values are measured in the outer regions of other BCGs, e.g. NGC 3557 (Brough et al. 2007), NGC 1400 (Spolaor et al. 2008), NGC 1407 (Spolaor et al. 2008; Forbes et al. 2011). But, contrarily to NGC 3311, the velocity dispersion profiles in the halos of these galaxies are either decreasing or nearly constant, indicating that the stars are still bound to the galaxy and that the contribution from intracluster stars to the kinematics is small. The stellar halo around NGC 3311, however, consists of mostly intracluster stars. The lack of a significant metallicity gradient over the spatial range probed by slit sections A-C indicates that the stars accumulated on the halo through several accretion episodes. What are the progenitors of these stars?

1. Stars from the central regions of massive early type galaxies are most likely ruled out. The distribution of their $[\mathrm{Z} / \mathrm{H}]$ $([\alpha / \mathrm{Fe}])$ has mean $0.35(0.19)$ and dispersion $0.30(0.07)$ in solar units. These average values are based on measurements in early-type galaxies in the Coma cluster with velocity dispersion $\sigma>200 \mathrm{~km} \mathrm{~s}^{-1}$, from Trager et al. (2008). The stellar population properties measured in the central regions of the early-type galaxies in Hydra I where measurements of the Lick indices are available (IC 2586, IC 2597, NGC 3308, ESO436-G04, and ESO501-G13) ${ }^{4}$ are consistent with the average values in Coma.

2. Stars in early-type galaxies outside 1-2 effective radii are potential candidates. Indeed, several early-type galaxies in the sample of Spolaor et al. (2010), Pu et al. (2010) have at $1-2$ effective radii $[\mathrm{Z} / \mathrm{H}]$ and $[\alpha / \mathrm{Fe}]$ similar to what we observe in the halo of NGC 3311. Unfortunately, no data outside of one effective radius are available for early-type galaxies in Hydra I, but only central values for a number of them (NGC 3305, NGC 3309, ESO436-G45 and ESO501-G13) ${ }^{4}$. They are characterized by alpha enhanced stars $([\alpha / \mathrm{Fe}]>0.4)$ at their centers, but they have $[\mathrm{Z} / \mathrm{H}]>0$. Stars stripped from their halos might be consistent with the stellar population of the stars around NGC 3311 under the (plausible) assumption that these galaxies are characterized by strong and negative metallicity gradients, and flat $[\alpha / \mathrm{Fe}]$ radial profiles.

3. Stars stripped from the disks or the bulges of late-type spirals are most likely ruled out. They are generally characterized by younger ages and by solar values of metallicity and $\alpha$-elements abundance (Crowl \& Kenney 2008; Morelli et al. 2008). The stellar population properties measured in the central regions of the late-type galaxies in Hydra I where measurements of the Lick indices are available (ESO437G015, ESO437-G45, and ESO501-G03) ${ }^{4}$ are consistent with these values. A small fraction in mass of young stars would decrease the luminosity-weighted age of the corresponding

\footnotetext{
${ }^{4}$ See Jorgensen (1997); Trager et al. (1998); Ogando et al. (2008) for the line strength of the Lick indices.
}

single stellar population (Serra \& Trager 2007), in contrast with our measurements.

4. Stars from disrupted dwarf galaxies are potential candidates. They are characterized on average by subsolar metallicity and nearly solar $\alpha$-enhancement; e.g., in the sample of Michielsen et al. (2008), the distribution of $[\mathrm{Z} / \mathrm{H}]([\alpha / \mathrm{Fe}])$ in dwarfs has mean value $-0.4(0.0)$ with a scatter of 0.3 (0.2) dex. Therefore, stars from disrupted dwarf galaxies can lower the luminosity weighted metallicity of the equivalent single stellar population. On the other hand, their contribution would also decrease the luminosity-weighted $[\alpha / \mathrm{Fe}]$, meaning that they cannot represent the main bulk of stars in the NGC 3311 halo.

In conclusion, the combination of kinematic and stellar population data suggests that the hot stellar halo around NGC 3311 was accreted. The bulk of the stars most likely originated from the outer regions of early-type galaxies with metal poor and $\alpha$-enhanced stellar populations. Stars from disrupted dwarf galaxies are also potential contributors, because of their generally low $[\mathrm{Z} / \mathrm{H}]$.

\subsection{The origin of the excess of light}

As discussed above, we have evidence that the stellar population measured in the long-slit bins D-E is different from that measured in bins A-C. Following the same dynamical and photometric arguments as in Sect. 4.1, we consider the contribution of stars bound to NGC 3311 to be small, but not the contribution of the excess of light. Therefore, there are potentially two main stellar populations in this region, and the approximation of single stellar population adopted in Sect. 3.1 may not be the appropriate way to interpret the observations.

Despite of the $S / N \geq 25$ (per pixel) reached and the good quality of the spectra, we have been unable to separate the two populations by simultaneously fitting their kinematics and stellar populations (e.g., using a direct spectral fit as in Coccato et al. 2011). This is probably because of the high velocity dispersion that broadens and mixes the spectral features of the two components along the line of sight.

A better way to interpret our observations is therefore to make some assumptions on the kinematics of the excess of light and on the nature of its progenitors on the basis of available additional information. Following these assumptions, we created a simulated spectrum $S_{\text {model }}$ from the combined contribution of the stellar halo and the excess of light and tested whether it is consistent with what is observed.

Arnaboldi et al. (2011) were able to estimate the kinematics of the excess of light from the cross correlation between a template star and a model spectrum for the excess. They found two positive peaks on the correlation function above $\sim 2 \sigma$ noise. The first peak was at $5032 \mathrm{~km} \mathrm{~s}^{-1}$, and it was interpreted as the velocity of the excess of light along the line of sight. The second was at $3905 \mathrm{~km} \mathrm{~s}^{-1}$, consistent with the systemic velocity of NGC 3311 and it was interpreted as the residual contamination of the stars from the halo in the model spectrum of the excess of light. Therefore the relative velocity of the excess of light with respect to NGC 3311 is on the order of $1200 \mathrm{~km} \mathrm{~s}^{-1}$. Arnaboldi et al. (2011) also propose a scenario in which the excess of light originates from disrupted dwarf galaxies and they estimate that the excess of light represents $10-20 \%$ of the surface brightness of the halo of NGC 3311 in the region where the excess is detected. 
Following their interpretation, we assume that we observe the superimposition of the halo stellar population and a dwarfgalaxy-like population in bins D-E. As template for the halo spectra, we used that extracted from bin A; as template for a dwarf galaxy spectrum we used that of the dwarf galaxy HCC 26, on which the FORS2 long-slit was centered (see the orange circle in Fig. 1). The stellar population properties of HCC26 are consistent with those of other dwarf galaxies (Michielsen et al. 2008). As a first approximation, we normalized the spectra of bin A and HCC 26 to their continuum values at $5100 \AA$. We indicate these normalized spectra with $S_{\text {halo }}$ and $S_{\text {dwarf }}$, respectively. We then convolved $S_{\text {dwarf }}$ with a Gaussian LOSVD to construct the contaminant component $\tilde{S}_{\text {dwarf }}$. The LOSVD we used for the convolution has mean velocity $1200 \mathrm{~km} \mathrm{~s}^{-1}$ higher than the systemic velocity of NGC 3311 and velocity dispersion of $450 \mathrm{~km} \mathrm{~s}^{-1}$. These assumptions are consistent with the estimate of the velocity of the excess of light with respect the halo around NGC 3311 (Arnaboldi et al. 2011), the typical velocity dispersion measured in these regions (Ventimiglia et al. 2010) and the standard deviation of the lineof-sight velocities of the seven dwarf galaxies located on the top of the excess of light $\left(\sigma_{7}\right.$ DWARFs $\sim 470 \mathrm{~km} \mathrm{~s}^{-1}$, Misgeld et al. 2008), which are potential progenitors. ${ }^{5}$ We then constructed many combined spectra $S_{\text {model }}=(1-F) \cdot S_{\text {halo }}+F \cdot \tilde{S}_{\text {dwarf }}$ for different values of the "contaminating" fraction $F$, measured their equivalent single stellar population values of age, $[\mathrm{Z} / \mathrm{H}]$ and $[\alpha / \mathrm{Fe}]$, and compared them with the observed stellar population properties in bins D and E. We prefer this strategy over a direct comparison of the observed and combined spectra $S_{\text {model }}$ because it is less sensitive to the uncertainties of the overall shape of the stellar continuum. Lick indices are less affected by these uncertainties, because the pseudo-continua and the pseudo-bands where each index is defined are very close in wavelength.

Figure 4 shows the results as function of $F$. The mean values of the single stellar population parameters measured in bins D-E are consistent with those of a composite population made of stars from the stellar halo around NGC 3311 and stars from disrupted dwarf galaxies. In bin $\mathrm{E}$ the contamination is stronger than in bin $\mathrm{D}$. In detail, the range of contamination in the surface brightness from $17 \%$ to $28 \%$ reproduces within uncertainties the mean values of age, $[\mathrm{Z} / \mathrm{H}]$ and $[\alpha / \mathrm{Fe}]$ measured in bin $\mathrm{E}$ (between horizontal green long-dashed lines). A smaller fraction $(<13 \%)$ is needed to explain the mean values observed in bin D (between horizontal magenta short-dashed lines). The ranges of contamination in surface brightness we found are on the same order of magnitude of the estimate in Arnaboldi et al. (2011).

The presence of a contaminant component with lower velocity dispersion $\left(\sigma \lesssim 60 \mathrm{~km} \mathrm{~s}^{-1}\right)$ was also tested, and we found that it can contribute less than $F \leq 5 \%$ to the local surface brightness in all bins. Higher fractions would produce absorption lines features clearly visible on the combined spectrum (contrarily to what we observe in bins D-E), which would be recovered by a direct spectral fit (Coccato et al. 2011).

Despite of the uncertainties on the kinematics that we assumed for the contaminant stellar component, this simple approach supports the idea that the excess of light in the Hydra I cluster core consists of stars stripped from dwarf galaxies, which are thus being added to the dynamically hot stellar halo around NGC 3311.

\footnotetext{
5 The results do not vary too much over the velocity dispersion range $200 \mathrm{~km} \mathrm{~s}^{-1} \lesssim \sigma \lesssim 600 \mathrm{~km} \mathrm{~s}^{-1}$, meaning that we cannot infer precise constraints on the kinematics using the stellar population properties.
}

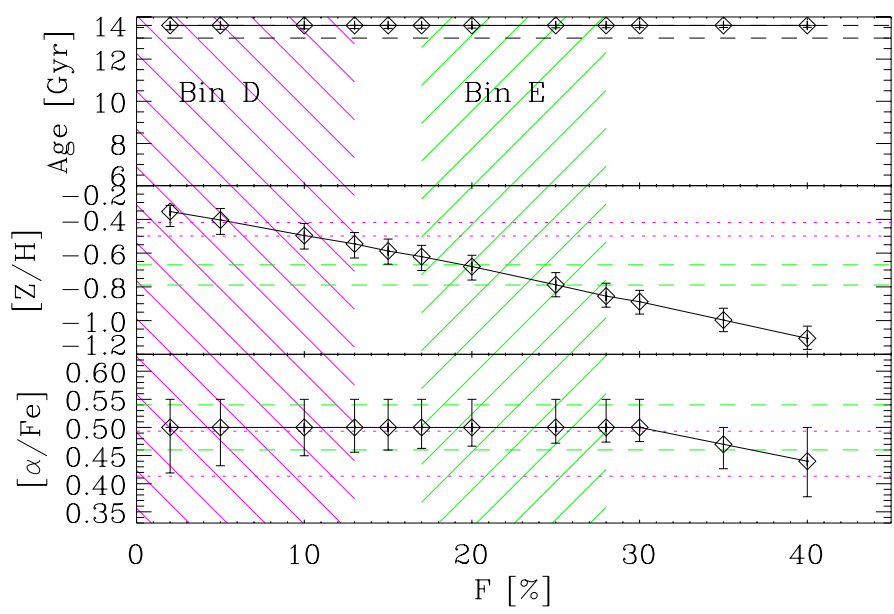

Fig. 4. Equivalent single stellar population age (upper panel), metallicity (central panel) and $\alpha$-enhancement (lower panel) measured in $S_{\text {model }} . S_{\text {model }}$ represent a composite stellar population formed by stars from the halo around NGC 3311 contaminated by a fraction $F$ of stars from dwarf galaxies (see Sect. 4.2 for details). Long-dashed green and short-dashed magenta lines represent the mean values of age, $[\mathrm{Z} / \mathrm{H}]$ and $[\alpha / \mathrm{Fe}]$ measured in the long-slit bins $\mathrm{E}$ and $\mathrm{D}$, respectively. The range of $F$ values consistent with measurements in bins $\mathrm{E}$ and $\mathrm{D}$ are shaded in green and magenta, respectively.

\section{Summary}

We used deep long-slit spectra obtained with FORS2 at the VLT on the northeast side of the brightest cluster galaxy NGC 3311 to study the stellar population content in five regions from $\sim 10$ to $\sim 30 \mathrm{kpc}$ from its center (slit sections A-E), where the contribution from intra-cluster stars dominates (Ventimiglia et al. 2010).

We found that the stars of the dynamically hot halo around NGC 3311 are on average characterized by an old, metal-poor and $\alpha$-enhanced stellar population. The lack of a stellar population gradient in slit sections $\mathrm{A}-\mathrm{C}$ together with the kinematics suggest that the intracluster halo formed from accretion of multiple components onto the cluster center. The stellar population parameters suggest that the halo stars were mainly stars stripped from the outer regions of early-type galaxies, with a possible contribution from dwarf galaxies.

In the long-slit sections D-E the metallicity is significantly lower than that measured in sections A-C. We associate this spectroscopic feature with the presence of a photometric substructure (excess of light), which was detected in the same area of the halo around NGC 3311 (Arnaboldi et al. 2011). Simulations of the measurements over this region are consistent with the presence of a composite population made of stars from the stellar halo around NGC 3311 and stars from disrupted dwarf galaxies, which form part of the excess of light. Our findings therefore support the idea that the build-up of ICL in the Hydra I cluster core and the stellar halo around NGC 3311 are still ongoing.

Acknowledgements. The authors wish to thank E. Iodice, H. Kuntschner, and L. Morelli for useful discussions.

\section{References}

Abadi, M. G., Navarro, J. F., \& Steinmetz, M. 2006, MNRAS, 365, 747 Appenzeller, I., Fricke, K., Fürtig, W., et al. 1998, The Messenger, 94, 1 Arnaboldi, M., Ventimiglia, G., Iodice, E., Gerhard, O., \& Coccato, L. 2011, A\&A, submitted

Arnold, J. A., Romanowsky, A. J., Brodie, J. P., et al. 2011, ApJ, 736, L26 
Bernardi, M., Roche, N., Shankar, F., \& Sheth, R. K. 2011, MNRAS, 412, L6 Brough, S., Proctor, R., Forbes, D. A., et al. 2007, MNRAS, 378, 1507 Cappellari, M., \& Emsellem, E. 2004, PASP, 116, 138

Cardiel, N., Gorgas, J., Cenarro, J., \& Gonzalez, J. J. 1998, A\&AS, 127, 597

Coccato, L., Arnaboldi, M., Gerhard, O., et al. 2010a, A\&A, 519, A95

Coccato, L. Gerhard, O., \& Arnaboldi, M. 2010b, MNRAS, 407, L26

damien.segransan@unige.ch Coccato, L., Morelli, L., Corsini, E. M., et al. 2011, MNRAS, 412, L113

Crowl, H. H., \& Kenney, J. D. P. 2008, AJ, 136, 1623

Davies, R. I. 2007, MNRAS, 375, 1099

De Lucia, G., \& Blaizot, J. 2007, MNRAS, 375, 2

Forbes, D. A., Spitler, L. R., Strader, J., et al. 2011, MNRAS, 413, 2943

Gerhard, O., Arnaboldi, M., Freeman, K. C., et al. 2007, A\&A, 468, 815

Gnedin, O. Y. 2003, ApJ, 589, 752

Hau, G. K. T., Hilker, M., Bridges, T., et al. 2004, in Outskirts of Galaxy Clusters: Intense Life in the Suburbs, ed. A. Diaferio, IAU Colloq., 195, 491 Jorgensen, I. 1997, MNRAS, 288, 161

Kapferer, W., Schindler, S., Knollmann, S. R., \& van Kampen, E. 2010, A\&A, 516, A41

Krick, J. E., Bernstein, R. A., \& Pimbblet, K. A. 2006, AJ, 131, 168

Kuntschner, H. 2004, A\&A, 426, 737

Loubser, S. I., Sansom, A. E., Sánchez-Blázquez, P., Soechting, I. K., \& Bromage, G. E. 2008, MNRAS, 391, 1009

Michielsen, D., Boselli, A., Conselice, C. J., et al. 2008, MNRAS, 385, 1374

Misgeld, I., Mieske, S., \& Hilker, M. 2008, A\&A, 486, 697

Moore, B., Katz, N., Lake, G., Dressler, A., \& Oemler, A. 1996, Nature, 379, 613

Morelli, L., Pompei, E., Pizzella, A., et al. 2008, MNRAS, 389, 341

Murante, G., Giovalli, M., Gerhard, O., et al. 2007, MNRAS, 377, 2

Naab, T., Johansson, P. H., \& Ostriker, J. P. 2009, ApJ, 699, L178
Ogando, R. L. C., Maia, M. A. G., Pellegrini, P. S., \& da Costa, L. N. 2008, AJ, 135,2424

Oser, L., Ostriker, J. P., Naab, T., Johansson, P. H., \& Burkert, A. 2010, ApJ, 725,2312

Pu, S. B., Saglia, R. P., Fabricius, M. H., et al. 2010, A\&A, 516, A4

Puchwein, E., Springel, V., Sijacki, D., \& Dolag, K. 2010, MNRAS, 406, 936

Richtler, T., Salinas, R., Misgeld, I., et al. 2011, A\&A, 531, A119

Rudick, C. S., Mihos, J. C., \& McBride, C. 2006, ApJ, 648, 936

Rudick, C. S., Mihos, J. C., Frey, L. H., \& McBride, C. K. 2009, ApJ, 699, 1518

Sánchez-Blázquez, P., Peletier, R. F., Jiménez-Vicente, J., et al. 2006, MNRAS, 371,703

Serra, P., \& Trager, S. C. 2007, MNRAS, 374, 769

Spolaor, M., Forbes, D. A., Proctor, R. N., Hau, G. K. T., \& Brough, S. 2008, MNRAS, 385, 675

Spolaor, M., Kobayashi, C., Forbes, D. A., Couch, W. J., \& Hau, G. K. T. 2010, MNRAS, 408, 272

Thomas, D., Maraston, C. \& Johansson, J. 2011, MNRAS, 412, 2183

Toledo, I., Melnick, J., Selman, F., et al. 2011, MNRAS, 414, 602

Trager, S. C., Worthey, G., Faber, S. M., Burstein, D., \& Gonzalez, J. J. 1998, ApJS, 116, 1

Trager, S. C., Faber, S. M., \& Dressler, A. 2008, MNRAS, 386, 715

Trujillo, I., Conselice, C. J., Bundy, K., et al. 2007, MNRAS, 382, 109

Trujillo, I., Ferreras, I., \& de La Rosa, I. G. 2011, MNRAS, 415, 3903

van Dokkum, P. G., Whitaker, K. E., Brammer, G., et al. 2010, ApJ, 709, 1018

Ventimiglia, G., Gerhard, O., Arnaboldi, M., \& Coccato, L. 2010, A\&A, 520, L9

Ventimiglia, G., Arnaboldi, M., \& Gerhard, O. 2011, A\&A, 528, A24

Willman, B., Governato, F., Wadsley, J., \& Quinn, T. 2004, MNRAS, 355, 159

Williams, B. F., Ciardullo, R., Durrell, P. R., et al. 2007, ApJ, 656, 756

Worthey, G., \& Ottaviani, D. L. 1997, ApJS, 111, 377

Worthey, G., Faber, S. M., Gonzalez, J. J., \& Burstein, D. 1994, ApJS, 94, 687 